\title{
Serum-Harnsäure-Werte von 113 Patienten mit Zustand nach Herzinfarkt
}

\author{
Von D. Sustć, A. Büumer, und D. Schultz \\ Aus dem Institut für Humangenetik (Direktor: Prof. Dr. Dr. b. c. W. Lenq) und der Medizinischen Klinik \\ (Direktor: Prof. Dr. W. H. Hauss) der Universität Münster
}

(Eingcgangen am 15. November 1968)

Bei 113 Infarktpatienten wurden die Serumharnsäurewerte untersucht. $48,8 \%$ hatten eine Hyperurikämie von mchr als $6,4 \mathrm{mg} / 100 \mathrm{~m} l$. Dic Verteilungskurve zeigt ein trimodales Verhalten und eine signifikante Abweichung vom theoretisch crmittelten Kurvenverlauf. Möglicherweise licgt ein genetischer Polymorphismus vor, der Beziehungen zur Infarktgencse hat.

\section{Serum uric acid values from 113 patients after beart infarct}

Uric acid levels were measured in the sera of 113 patients with heart infarct. $48.8 \%$ had a hyperuricaemia of more that $6.4 \mathrm{mg} / 100 \mathrm{ml}$. The distribution curve showed a trimodal behaviour and a significant deviation from the theoretical curve. There possibly exists a polymorphism, which has a relationship to the genesis of infarct.

Auf die Zusammenhänge zwischen Hyperurikämie und Koronarkrankheiten wurde in neuerer Zeit mehrfach hingewiesen $(1,2,3)$. So bezeichnet HeNCH den Herzinfarkt neben der Apoplexie als eine der Todesursachen bei Gichtkranken (4). In Arterien, Endokard, Herzklappen, Herzmuskel sowie in Infarktzonen wurden Uratkristalle gefunden $(5,6,7,8)$. Es schien deshalb sinnvoll, die Serumharnsäure bei Patienten mit Zustand nach einem Herzinfarkt zu bestimmen.

\section{Methodik}

\section{Patienten}

Es wurden 113 Männer im Alter zwischen 37 und 63 Jahren (Durchschnittsalter 49 Jahre) untersucht. Beim größten Teil von ihnen lag zur Zeit der Untersuchung der Herzinfarkt 4 Monate bis 21/2 Jahre (durchschnittlich $1 \mathrm{Jahr}$ ) zurück. Alle Patienten befanden sich in einer Kurklinik. 30\% von ihnen erhielten kalorienarme Kost (1200 bzw. 1700 Kalorien), die anderen, abgesehen von 2 Diabetikern, Vollkost. Je nach Besonderheit des Krankheitsbildes wurden sic mit Koronardilatatoren, Nitropräparaten, kreislaufunterstützenden und blutdrucksenkenden Mitteln behandelt und digitalisiert. Die beiden Zuckerkranken bekamen orale Antidiabetika.

\section{Harnsäurebestimmung}

Die Blutentnahme erfolgte nüchtern durchschnittlich 22 Tage nach der Klinikaufnahme. Die Serumharnsäure wurde mit einem automatischen Analysensystem (Technicon-Autoanalysator) nach dem Folin-Wu-Prinzip in einem Arbeitsgang bestimmt und die Analysencrgebnisse durch pericdisch eingeschaltete Kontrollseren bzw. Eich- und Standardlösungen regelmäßig geprüft. Diesc Methode bat eine gute Wiederholbarkeit (Variationskoeffizient $\mathrm{V}= \pm 2,2 \%$ ) (9).

\section{Ergebnisse}

Der arithmetische Mittelwert der Serumharnsäurekonzentration von 113 Infarktpatienten beträgt $6,4 \mathrm{mg} /$ $100 \mathrm{~m} /$ (bei 181 Gelegenheitsblutspendern $6,1 \mathrm{mg} /$ $100 \mathrm{~m} l$ (10). Das in der Abbildung 1a dargestellte Diagramm zeigt eine trimodale Verteilung der Serumharnsäurewerte bei Infarktpatienten, gegenüber einer bimodalen Verteilung bei Gelegenheitsblutspendern. Die Einzelwerte sind in der Tabelle 1 aufgeführt. Die kumulative Frequenz und der Kurvenverlauf der beobachteten Werte weichen signifikant von der theoretisch ermittelten Verteilung ab (geprüft nach dem Kolmogorov-Smirnov-Test) (Tab. 1, Abb. 1 b).

\section{Diskussion}

Nimmt man als oberen Normalwert der Serumharnsäure $6,4 \mathrm{mg} / 100 \mathrm{~m} /$ (die Konzentration, bei der die

Tab. 1

Die beobachtete und theoretisch errechnete Verteilung und kumulative Frequenz der Serumhanrsäurewerte von 113 Patienten mit Zustand nach Herzinfarkt

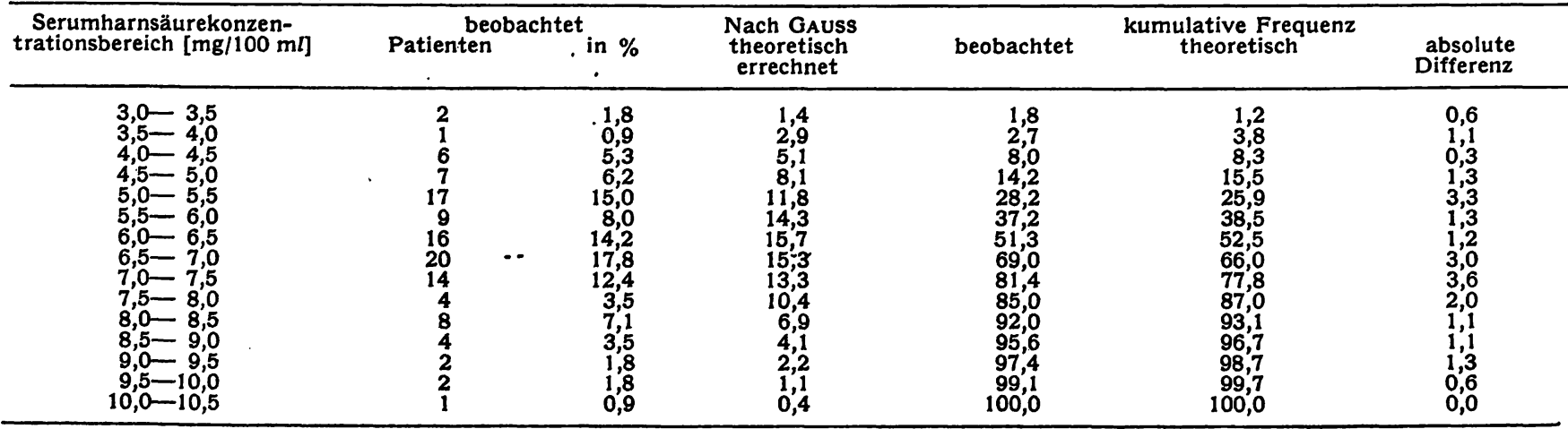




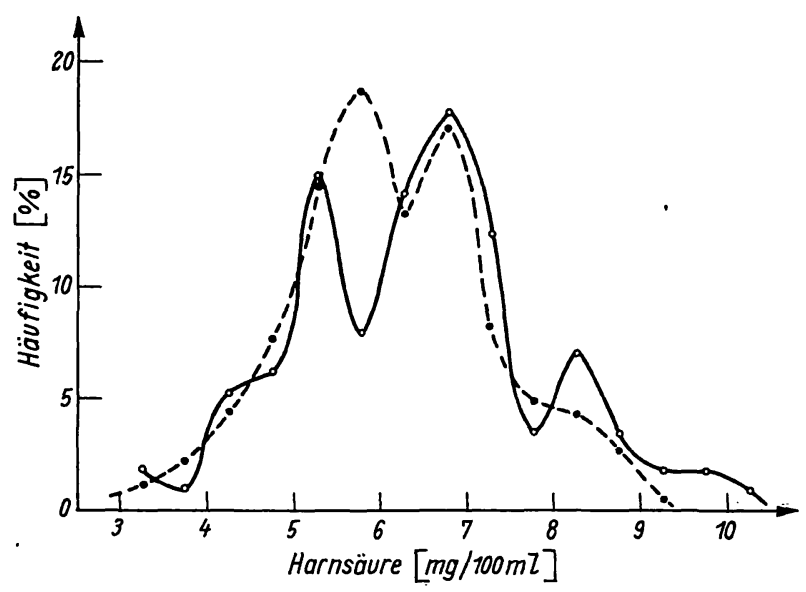

Abb. 1 a

Die Verteilungskurve der Serumharnsäurewerte bei Infarktpatienten $(-)$, im Vergleich zu der von Gelegenheitsblutspendern (-------)

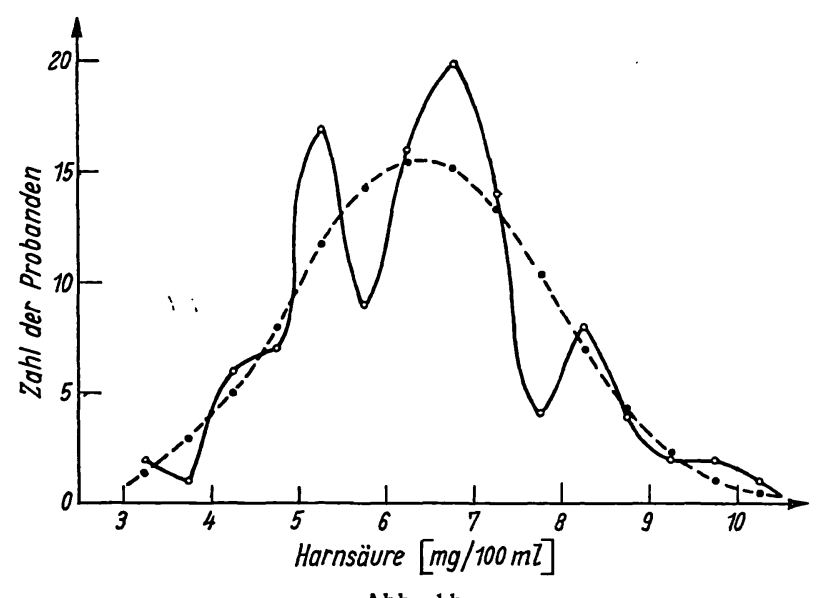

Abb. 1 b

Die Verteilungskurve der Serumharnsäurewerte bei 113 Infarktpatienten $(\longrightarrow$ ) im Vergleich zur errechneten GAUss-Verteilung
Serumharnsäure je nach individuellem pH-Wert und $\mathrm{Na}^{+}-$Haushalt des Blutes zu Mononatriumurat-Monohydrat ausfällt) an $(11,12)$, haben $48,8 \%$ der Patienten eine Hyperurikämie. Hiermit stimmen auch die Ergebnisse anderer Untersucher überein $(13,14,15)$. Wir haben bereits gezeigt, daß homogene Untersuchungsgruppen der gesunden Bevölkerung eine bimodale Verteilung der Serumharnsäure aufweisen (10). Die Zweigipflichkeit der Kurve kann genetisch oder pathophysiologisch bedingt sein. Die Verteilungskurve der Serumharnsäurewerte bei Infarktpatienten zeigt einen trimodalen Verlauf mit stark ausgeprägtem zweiten Gipfel. Der dritte Gipfel im Bereich zwischen 7,5 und $9,0 \mathrm{mg} / 100 \mathrm{~m} l$ war schon bei den Gelegenheitsblutspendern angedeutet (Abb. 1a). So fanden auch DECKER, LANE und REYNoLDs (16) bei 118 Filipinos eine Harnsäureverteilung, die auf zwei verschiedene Kollektive hinweist, die sich im Sinne eines genetischen Polymorphismus hinsichtlich ihrer endogenen Harnsäureproduktion unterscheiden könnten. Dafür könnten auch neuere Befunde sprechen, die eine Enzymopenie der Hypoxanthin-Phosphoribosyltransferase ${ }^{1}$ ) bei Verwandten von Gichtkranken nachwiesen (17). Die Hypoxanthin-Phosphoribosyltransferase ist für die Harnșāureproduktion mitverantwortlich.

Die Arbeit wurde in dankenswerter Weise durch das Lańdesamt für Forschung des Landes Nordrhein-Westfalen unterstützt.

Wir möchten Herrn Prof. Dr. Immo von Hatringberg und den Oberärzten Dr. Herbert Mensen und Dr. Hans Engel ining von dem Sanatorium „Teutoburger Wald" in Bad Rothenfelde für die freundliche Uberlassung der Blutproben danken.

1) IMP: Pyrophosphat Phosphoribosyltransferase (EC 2.4.2.8)

\section{Literatur}

1. Gertler, M. M., Lancet, London 1963/I, 1054. - 2. GertLer, M. M., M. A. Woodbury, L. G. GotsCh, P. D. Whrte und H. A. Rusk, J. . Amer. Med. Ass. 170, 149 (1959). - 3. HaLL, A. R., Arthritis Rheum: 8, 846 (1965). - 4. HENCH, P. S., zit. n. LöFFLER, W. und F. Koller, Handbuch der Inneren Medizin, 4. Aufl., 7. Bd. II. Teil, S. 491 Springer-Verlag Berlin-Göttingen-Heidelberg (1955). - 5. PAullex, J. W., Lancet, London 1962/II, 1273. 6. Talbotr, J. H., Gout, Grune \& Stratton Verlag New York/ London (1957). - 7. LöFFleR, W. und F. KolleR, Handbuch der Inneren Medizin, 4. Auf., 7. Bd., II. Teil, S. 491 SpringerVerlag Berlin-Göttingen-Heidelberg (1955). - 8. KuzELL, W. C. und G. P. Gaudin, Gicht, Documenta theumat. (Basel) 10 (1956). - 9. Eggstein, M., R. Allmer, W. KNodel und E. KuhlMANN, Automation in der Analytischen Chemie (1965), Technicon
GmbH, Frankfurt 591-604 (1966). - 10. Susić, D. und A. BäUmer, Z. Rheumaforsch., Im Druck. - 11. Peters, J. P. und D. D. van SLYKE, Quantitative Clinical Chemistry, Interpretations, S. 950, Williams and Wilkins, Baltimore (1946). - 12. GudzENT, F., Hoppe-Seỳler, Z. physiol. Chem. 60, 25 (1909). - 13. SAŔ KADY, L., Klin. Wschr. 21, 884 (1952). - 14. GertLer, M. M., S. M. GARN und S. A. Levine, Ann. Int. Med. 34, 1421 (1951). - 15. London, M. und M. Hưss, Clin. Chem. New York 13, 132 (1967). - 16. Decker, J. L., J. J. LANe und W. E. Reynolds, Arthritis Rheum. 5, 144 (1962). - 17. GreENE, M. L., W. Y. Fujrmoto, I. M. Rosenthal und J. E. SeEgmrller, Abstracts of the American Society of Human Genetics, Austin, Texas, October 10-13, 1968.
Dipl. Ing. Chem. Dražen Susić Institut für Humangenetik 44 Münster Vesaliușweg 12/14 